\title{
Electromagnetic contributions to pseudoscalar masses
}

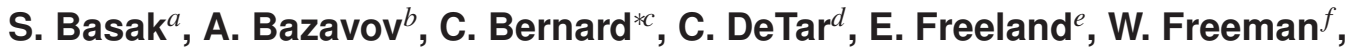 J. Foley ${ }^{d}$, Steven Gottlieb ${ }^{g}$, U.M. Heller ${ }^{h}$, J.E. Hetrick ${ }^{i}$, J. Laiho $^{j, k}$, L. Levkova ${ }^{l, d}$, M. Oktay ${ }^{d}$, J. Osborn ${ }^{m}$, R.L. Sugar ${ }^{n}$, A. Torok ${ }^{g}$, D. Toussaint ${ }^{l}$, R.S. Van de Water ${ }^{o, b}$, R. Zhou ${ }^{g}$ (MILC Collaboration)}

a NISER, Bhubaneswar, Orissa 751005, India

${ }^{b}$ Physics Department, Brookhaven National Laboratory, Upton, NY 11973, USA

${ }^{c}$ Department of Physics, Washington University, St. Louis, MO 63130, USA

${ }^{d}$ Department of Physics and Astronomy, University of Utah, Salt Lake City, UT 84112, USA

${ }^{e}$ Department of Physics, Benedictine University, Lisle, IL 60532, USA

${ }^{f}$ Department of Physics, George Washington University, Washington, DC 20037, USA

${ }^{g}$ Department of Physics, Indiana University, Bloomington, IN 47405, USA

${ }^{h}$ American Physical Society, One Research Road, Ridge, NY 11961, USA

${ }^{i}$ Physics Department, University of the Pacific, Stockton, CA 95211, USA

${ }^{j}$ SUPA, School of Physics and Astronomy, University of Glasgow, Glasgow, UK

${ }^{k}$ Department of Physics, Syracuse University, Syracuse, NY 13244, USA

${ }^{l}$ Physics Department, University of Arizona Tucson, AZ 85721, USA

${ }^{m}$ ALCF, Argonne National Laboratory, Argonne, IL 60439, USA

${ }^{n}$ Physics Department, University of California, Santa Barbara, CA 93106, USA

o Theoretical Physics Department, Fermi National Accelerator Laboratory, Batavia 60510, USA

E-mail: cb@wust l.edu

\begin{abstract}
We report on the calculation by the MILC Collaboration of the electromagnetic effects on kaon and pion masses. These masses are computed in QCD with dynamical (asqtad staggered) quarks plus quenched photons at three lattice spacings varying from 0.12 to $0.06 \mathrm{fm}$. The masses are fit to staggered chiral perturbation theory with NLO electromagnetic terms, as well as analytic terms at higher order. We extrapolate the results to physical light-quark masses and to the continuum limit. At the current stage of the analysis, most, but not all, of the systematic errors have been estimated. The main goal is the comparison of kaon electromagnetic splittings to those of the pion, i.e., an evaluation of the corrections to "Dashen's theorem." This in turn will allow us to significantly reduce the systematic errors in our determination of $m_{u} / m_{d}$.
\end{abstract}

The 7th International Workshop on Chiral Dynamics,

August 6-10, 2012

Jefferson Lab, Newport News, Virginia, USA

\footnotetext{
${ }^{*}$ Speaker.
} 
Introduction. - The disentangling of electromagnetic (EM) and isospin-violating effects in the kaon and pion systems is a long-standing problem. Understanding these effects is crucial for computing light quark masses, which are fundamental parameters in the Standard Model and important for phenomenology. Indeed, the size of the EM contributions to the kaon masses is the largest uncertainty in determinations of $m_{u} / m_{d}$ from the lattice [1], and in particular in our calculations [2]. The contributions have until recently been taken from a variety of phenomenological estimates, and therefore have quite large and not well controlled errors. We have been working on reducing these uncertainties for some time by calculating the EM effects directly on the lattice; progress has been reported previously in Refs. [3, 4].

The error in $m_{u} / m_{d}$ is dominated by the error in the mass difference $\left(M_{K^{ \pm}}^{2}-M_{K^{0}}^{2}\right)^{\gamma}$, where $\gamma$ denotes the total EM contribution, i.e., the difference between the value of a quantity in the presence of electromagnetism, and its value in a world in which all EM charges, both of valence and of sea quarks, are turned off. One may try to relate this difference to the much better understood difference in the pion system, $\left(M_{\pi^{ \pm}}^{2}-M_{\pi^{0}}^{2}\right)^{\gamma}$. To lowest order (LO) in chiral perturbation theory $(\chi \mathrm{PT})$ these EM splittings are in fact the same; this observation is known as Dashen's theorem [5]. We aim to calculate on the lattice the corrections to Dashen's theorem, which may be parameterized by

$$
\left(M_{K^{ \pm}}^{2}-M_{K^{0}}^{2}\right)^{\gamma}=(1+\varepsilon)\left(M_{\pi^{ \pm}}^{2}-M_{\pi^{0}}^{2}\right)^{\gamma} .
$$

Our computations employ full QCD but quenched photons. As pointed out in Ref. [6], however, the EM-quenching effects on $\varepsilon$ may be calculated and corrected to NLO in $\chi$ PT, with controlled errors. Similarly, squared mass differences of the form $\left(M_{P}^{2}-M_{P^{\prime}}^{2}\right)^{\gamma}$ are calculable with controlled errors in our setup, where $P$ is any light pseudoscalar meson and $P^{\prime}$ is the corresponding meson made from neutral valence quarks with the same masses as those in $P$. We also compute the EM effects on the $K^{0}$ alone, namely $\left(M_{K^{0}}^{2}\right)^{\gamma}$. In this case, however, the quenching effects are not calculable in $\chi \mathrm{PT}$, and uncontrolled errors remain. For $m_{u} / m_{d}$, the uncertainty coming from $\left(M_{K^{0}}^{2}\right)^{\gamma}$ is, fortunately, subdominant.

In the pion system, isospin-violating effects on the mass splitting are known to be small (see, e.g., Ref. [1]), so the experimental splitting is almost completely electromagnetic: $\left(M_{\pi^{ \pm}}^{2}-\right.$ $\left.M_{\pi^{0}}^{2}\right)^{\text {expt }} \approx\left(M_{\pi^{ \pm}}^{2}-M_{\pi^{0}}^{2}\right)^{\gamma}$. It would be costly to simulate the true $\pi^{0}$, which has EM disconnected diagrams even in the isospin limit. Instead, we simulate a " $\pi_{0}$ " whose squared mass is a simple average of the squared masses of $u \bar{u}$ and $d \bar{d}$ mesons, computed with connected diagrams only. Because all EM contributions to neutral mesons vanish in the chiral limit, both the true $\left(M_{\pi^{0}}^{2}\right)^{\gamma}$ and our $\left(M^{\prime \prime} \pi^{0},\right)^{\gamma}$ are small in any case. From Zweig-rule considerations, we suspect that the disconnected contribution is smaller still. We estimate

$$
\left(M_{\pi^{0}}^{2}\right)^{\gamma} \sim\left(M_{“ \pi^{0},}^{2}-M_{\pi^{\prime}}^{2}\right)^{\gamma}
$$

Since there are errors coming both from quenched electromagnetism and from the neglect of disconnected diagrams, one might expect $\sim 100 \%$ errors in this estimate, however. On the other hand, our calculation of the EM pion splitting, using $\left(M_{\pi^{ \pm}}^{2}-M_{\pi^{0}}^{2}\right)^{\gamma} \approx\left(M_{\pi^{ \pm}}^{2}-M_{*}^{2} \pi^{0},\right)^{\gamma}$ suffers only from the neglect of disconnected diagrams, not from uncontrolled quenching effects. As a rough estimate of the former we take $50 \%$ of $\left(M_{\pi^{0}}^{2}\right)^{\gamma}$, calculated through Eq. (2).

Chiral Perturbation Theory. - We fit our lattice data to expressions from (partially quenched) staggered chiral perturbation theory (S $\chi \mathrm{PT})$ in order to extrapolate to the physical light quark masses and to the continuum. We consider Goldstone (taste $\xi_{5}$ ) pseudoscalar mesons composed of 
valence quark $x$ and valence antiquark $y$, with masses $m_{x}$ and $m_{y}$. Let $M_{x y, 5}$ be the mass of such a meson with valence-quark charges $q_{x}$ and $q_{y}\left(q_{x y} \equiv q_{x}-q_{y}\right.$ is the meson charge), and let $\Delta M_{x y, 5}^{2}$ be the squared-mass splitting $\Delta M_{x y, 5}^{2} \equiv M_{x y, 5}^{2}-M_{x^{\prime} y^{\prime}, 5}^{2}$, where the primes in the second subscript indicate that the valence-quark charges are set to zero. To NLO in S $\chi \mathrm{PT}, \Delta M_{x y, 5}^{2}$ is given by [7]:

$$
\begin{gathered}
\Delta M_{x y, 5}^{2}=q_{x y}^{2} \delta_{E M}-\frac{1}{16 \pi^{2}} e^{2} q_{x y}^{2} \mathscr{M}_{x y, 5}^{2}\left[3 \ln \left(\mathscr{M}_{x y, 5}^{2} / \Lambda_{\chi}^{2}\right)-4\right] \\
-\frac{2 \delta_{E M}}{16 \pi^{2} f^{2}} \frac{1}{16} \sum_{\sigma, \xi}\left[q_{x \sigma} q_{x y} \mathscr{M}_{x \sigma, \xi}^{2} \ln \left(\mathscr{M}_{x \sigma, \xi}^{2}\right)-q_{y \sigma} q_{x y} \mathscr{M}_{y \sigma, \xi}^{2} \ln \left(\mathscr{M}_{y \sigma, \xi}^{2}\right)\right] \\
+c_{1} q_{x y}^{2} a^{2}+c_{2} q_{x y}^{2}\left(2 m_{l}+m_{s}\right)+c_{3}\left(q_{x}^{2}+q_{y}^{2}\right)\left(m_{x}+m_{y}\right)+c_{4} q_{x y}^{2}\left(m_{x}+m_{y}\right)+c_{5}\left(q_{x}^{2} m_{x}+q_{y}^{2} m_{y}\right)
\end{gathered}
$$

where $\delta_{E M}$ is a low-energy constant (LEC), $\sigma$ runs over the sea quarks, $\xi$ runs over the staggered tastes, $c_{i}$ are the LECs at NLO, and $m_{l}, m_{s}$ are the light and strange sea-quark masses. At this order, the meson masses denoted by $\mathscr{M}$ on the right hand side may be taken to be the tree-level masses in the absence of electromagnetism. Note that Ref. [7] mentions an additional NLO analytic term proportional to $\left(q_{x}^{2}+q_{y}^{2}\right) a^{2}$; this is not possible for a Goldstone splitting because it does not vanish as $m_{x}+m_{y}$ for small quark masses in the limit $q_{y}=q_{x}$, where EM effects are chirally symmetric.

Our statistical errors in $\Delta M_{x y, 5}^{2}$ are $\sim 0.3 \%$ for charged mesons and $\sim 1.0 \%$ for neutral mesons. It is clear that NLO S $\chi \mathrm{PT}$ cannot be expected to give a good description of the splittings at that level of precision. For reasonable fits, NNLO terms are needed. The S $\chi$ PT logarithms have not been calculated at that order, but we add all possible analytic terms. This may be justified by noting that the NNLO logarithms will be small at low mass, where the extrapolation is performed, and will be well approximated by analytic terms in the region near $m_{s}$.

To allow for finite-volume (FV) effects, we include standard terms dependent on $m_{\pi} L_{s}$ from EM tadpoles ( $L_{s}$ is the spatial lattice size), as well as an empirical EM finite-volume correction of the form $f_{v} q_{x y}^{2} / L_{s}^{2}$ used previously in Ref. [8], where $f_{v}$ is a constant. However, as discussed below, our measured finite-volume effects are rather small at present, and including or omitting the finite-volume terms from the fits makes little difference in the final results.

Lattice setup. - We calculate the meson spectrum in quenched EM backgrounds on a set of asqtad ensembles with $2+1$ flavors and $0.12 \mathrm{fm} \gtrsim a \gtrsim 0.06 \mathrm{fm}$. See Ref. [4] for a table of lattice parameters. The valence quarks have charges $\pm 2 / 3 e, \pm 1 / 3 e$ or 0 , where $e=e_{\text {phys }}, 2 e_{\text {phys }}$, or $3 e_{\text {phys }}$ ( $e_{\text {phys }}$ is the physical electron charge). For the results reported here, only the $e=e_{\text {phys }}$ data is used.

Figure 1 shows some of our partially quenched data for the splittings of physically charged $\pi^{ \pm}$ and $K^{ \pm}$mesons. We investigate the FV effects on two ensembles with $a \approx 0.12 \mathrm{fm}, a m_{l}=0.01$, and $a m_{s}=0.05$, but different volumes $\left(L \equiv L_{s} / a=20\right.$ and 28). The figure shows a comparison of the FV effect seen in our data with the difference between the two volumes expected from the results of the BMW Collaboration [8]. The effect we see is smaller: 0.35(45) of the difference expected from BMW. However, our larger-volume ensemble has only 274 configurations, leading to the rather large errors in the comparison. We are currently increasing the statistics on that ensemble in hopes of clarifying the issue.

We have measured the taste splitting between the Goldstone pion and the other local pion on our ensembles. The amount of taste violation caused by high-momentum photons is nonnegligible for mesons made from quarks with higher-than-physical charges, although it is always significantly smaller than that caused by high-momentum gluons. The fit function Eq. (3) is based on the neglect 
of taste violations caused by photons; that is the reason that we focus here only on the data with physical quark charges. Given that photon-induced taste violations are relatively small, however, one could expand the fit function in powers of $\alpha_{E M}=e^{2} /(4 \pi)$. Thus, inclusion of $\alpha_{E M}^{2}$ analytic terms to the fit function should allow the higher-charge data to be fit. That approach seems to work, and will be explored more in the future. For more details on EM taste-violating effects, see Ref. [4].

Results and Outlook. - Figure 2 shows a typical fit of our data for $\Delta M^{2}$ with physical quark charges to Eq. (3) (with added analytic NNLO terms). We fit partially quenched charged- and neutral-meson data simultaneously, but only the (unitary or approximately unitary) charged-meson data is shown in the plot. This fit has 55 data points and 26 parameters; other fits have as many as 120 data points, and from 20 to 30 fit parameters, depending on how many of the NNLO terms are included, and whether small variations with $a^{2}$ of the LO and NLO low-energy constants are allowed. The covariance matrix of the data is nearly singular, and the statistics are insufficient to determine it with enough precision to yield good correlated fits, so almost all fits currently used are uncorrelated. The fit shown has an (uncorrelated) $p$ value of 0.09 . We note that what appear to be big discretization effects are actually due in large part to mistunings of the strange-quark mass, which is off by about $50 \%$ on the $a=0.12 \mathrm{fm}$ ensembles and $25 \%$ on the $a=0.09$ fm ensembles, but only by $2 \%$ on the $0.06 \mathrm{fm}$ ensemble.

The black and brown lines in Fig. 2 show the fit after setting valence and sea masses equal, adjusting $m_{s}$ to its physical value, and extrapolating to the continuum. The black lines adjust the sea charges to their physical values using NLO $\chi$ PT, while the brown line keeps the sea quarks uncharged. In the pion case, the adjustment vanishes identically, so no brown line is visible. In

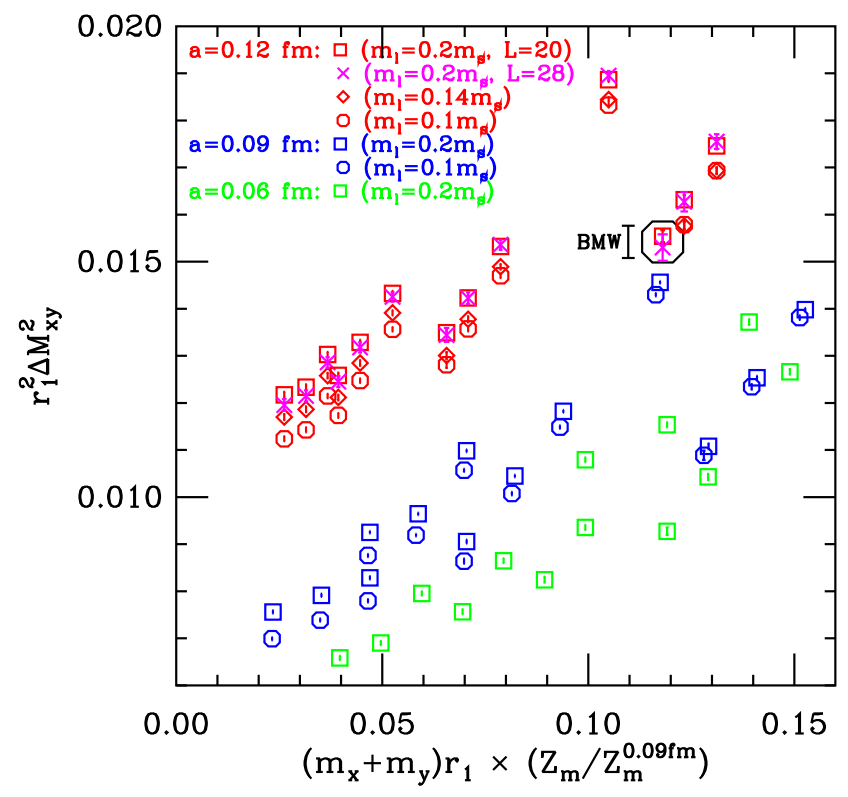

Figure 1: A sampling of our partially quenched data in $r_{1}$ units for EM splittings of pseudoscalar mesons with charge $\pm e_{\text {phys }}$, plotted versus the sum of the valence-quark masses. For clarity, only about a quarter of the data is shown. The red squares and magenta crosses show results for the two ensembles that differ only by the spatial volume: $20^{3}$ and $28^{3}$, respectively. The vertical black bar labeled "BMW" shows the expected difference for kaons between these two volumes, based on the results from the BMW collaboration [8]. Next to it, the two points encircled in black are our "kaon-like" points for the volumes. 


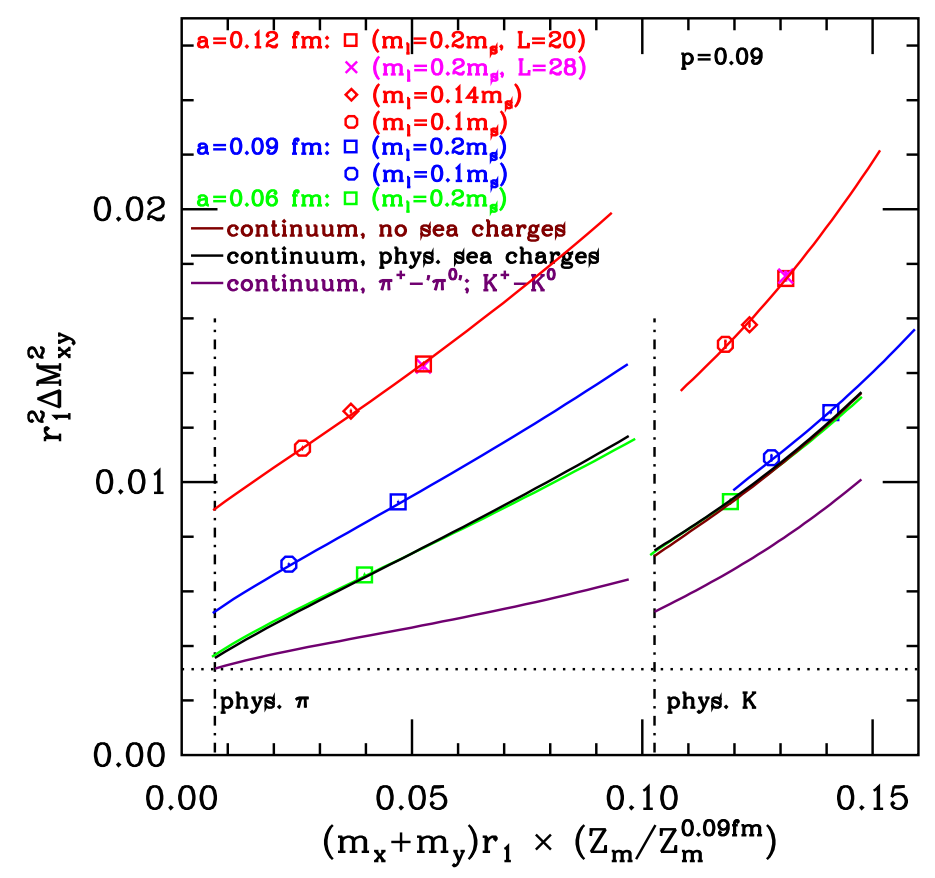

Figure 2: Typical S $\chi \mathrm{PT}$ fit to the squared-mass EM splitting $\Delta M^{2} v s$. the sum of the valence-quark masses. Only a small subset of the charged-meson data is shown. The red, blue and green curves correspond to three different lattice spacings. The brown and black curves are continuum limits for $\Delta M^{2}$, without or with the correction from $\chi \mathrm{PT}$ for physical sea-quark charges. The purple curves are the continuum limits for the $K^{+}-K^{0}$ splitting (right), and the $\pi^{+}-“ \pi^{0}$ " splitting (left).

the kaon case, the adjustment is a very small correction. From the black lines for the $\pi^{+}$and $K^{+}$, we subtract the corresponding results for the neutral mesons, " $\pi^{0}$ " and $K^{0}$, giving the purple lines. Results for $\left(M_{\pi^{+}}^{2}-M_{\text {“ } \pi^{0},}^{2},\right)^{\gamma}$ and $\left(M_{K^{+}}^{2}-M_{K^{0}}^{2}\right)^{\gamma}$ are then obtained from the intersections of the purple lines and the vertical dashed-dotted lines that give the location of the physical point for each meson. The excellent agreement of the result for $\left(M_{\pi^{+}}^{2}-M_{“{ }^{0} \pi^{0},}^{2}\right)^{\gamma}$ and the experimental pion splitting (horizontal dotted line) is accidental, since our result has roughly $20 \%$ total error.

We find the following preliminary results:

$$
\begin{array}{rlrl}
\left(M_{K^{+}}^{2}-M_{K^{0}}^{2}\right)^{\gamma} & =2100(90)(250) \mathrm{MeV}^{2}, & \left(M_{K^{0}}^{2}\right)^{\gamma}=901(8)(9)(?) \mathrm{MeV}^{2} \\
\left(M_{\pi^{+}}^{2}-M_{\left.“ \pi^{0},\right)^{\gamma}}^{2}\right. & =1270(90)(230)(80) \mathrm{MeV}^{2}, & \left(M_{“}^{2} \pi^{0,}\right)^{\gamma}=157.8(1.4)(1.7)(?) \mathrm{MeV}^{2} \\
\varepsilon & =0.65(7)(14)(10) & &
\end{array}
$$

The first two errors in each case are statistical and lattice systematic uncertainties. The latter error comes largely from the effects of changing the assumptions entering into the chiral/continuum fit. Note, however, that finite-volume errors are not included at present. We expect that ultimately they will be a significant, but subdominant, source of error. The "?" for $\left(M_{K^{0}}^{2}\right)^{\gamma}$ and $\left(M_{\text {“ } \pi^{0}}^{2} \text { " }\right)^{\gamma}$ represent the effect of EM quenching and, for $\left(M_{\text {" }}^{2} \pi^{0},\right)^{\gamma}$, the effect of neglected disconnected diagrams. These errors are likely to be much larger than the small quoted errors. For $\left(M_{\pi^{+}}^{2}-M_{\text {“" } \pi^{0},}^{2}\right)^{\gamma}$ and $\varepsilon$ the third error is a rough guess of the effect of neglecting disconnected diagrams, which we estimate by $50 \%$ of the result for $\left(M_{*}^{2} \pi^{0},\right)^{\gamma}$. If we redefine $\varepsilon$ by replacing our computation of the pion EM splitting in Eq. (1) with the experimental splitting, we get $\varepsilon=0.66(7)(20)$, which has larger chiral/continuum- 
extrapolation errors, but no error from neglecting disconnected contributions. Our result for $\varepsilon$ is compatible with results from other groups [9,8]. Using our values for $\varepsilon$ and $\left(M_{K^{0}}^{2}\right)^{\gamma}$ (assuming $100 \%$ EM-quenching error in the latter quantity), our preliminary estimate for the EM uncertainty in $m_{u} / m_{d}$ is reduced by approximately a factor of two [10] from our previous error [2].

We are currently finishing the analysis of two more-chiral ensembles at $a \approx 0.06 \mathrm{fm}$ in order to improve the chiral and continuum extrapolations, and are increasing the statistics for our finitevolume study. A "second-generation" calculation on HISQ ensembles, including ones at physical quark masses, is now beginning, and promises significant reductions in systematic errors. Calculations with dynamical EM effects included are also being contemplated.

Acknowledgments: We thank Laurent Lellouch and Taku Izubuchi for useful discussions. Computations for this work were carried out with resources provided by the USQCD Collaboration, the Argonne Leadership Computing Facility, and the National Energy Research Scientific Computing Center, which are funded by the Office of Science of the U.S. Department of Energy; and with resources provided by the National Center for Atmospheric Research, the National Institute for Computational Science, the Pittsburgh Supercomputer Center, the San Diego Supercomputer Center, and the Texas Advanced Computer Center, which are funded through the National Science Foundation's XSEDE Program. This work was supported in part by the U.S. Department of Energy under Grants DE-FG02-91ER- 40628, DE-FG02-91ER-40661, DE-FG02-04ER-41298, and DE-FC02-06ER41446; and by the National Science Foundation under Grants PHY07-57333, PHY07-03296, PHY07-57035, PHY07-04171, PHY09-03571, PHY09-70137, and PHY10-67881. This manuscript has been co-authored by an employee of Brookhaven Science Associates, LLC, under Contract No. DE-AC02-98CH10886 with the U.S. Department of Energy. Fermilab is operated by Fermi Research Alliance, LLC, under Contract No. DE-AC02-07CH11359 with the U.S. Department of Energy. For this work we employ QUDA [11].

\section{References}

[1] G. Colangelo, et al., Eur. Phys. J. C 71, 1695 (2011) [arXiv:1011.4408].

[2] A. Bazavov et al., Rev. Mod. Phys. 82, 1349 (2010) [arXiv:0903.3598]; A. Bazavov et al. [MILC], PoS LATTICE 2009, 079 (2009) [arXiv:0910.3618]; C. Aubin et al., [MILC], Phys. Rev. D 70, 114501 (2004) [hep-lat/0407028].

[3] S. Basak et al. [MILC], PoS LATTICE 2008, 127 (2008) [arXiv:0812.4486]; A. Torok et al., PoS LATTICE 2010, 127 (2010).

[4] S. Basak et al. [MILC], PoS LATTICE 2012, 137 (2012) [arXiv:1210.8157].

[5] R. Dashen, Phys. Rev. 183, 1245 (1969).

[6] J. Bijnens and N. Danielsson, Phys. Rev. D 75, 014505 (2007) [hep-lat/0610127].

[7] C. Bernard and E. D. Freeland, PoS LATTICE 2010, 084 (2010) [arXiv:1011.3994].

[8] A. Portelli et al., [BMW], PoS LATTICE 2011, 136 (2011) [arXiv:1201.2787] and PoS LATTICE 2010, 121 (2010) [arXiv:1011.4189].

[9] T. Blum et al., Phys. Rev. D 82, 094508 (2010) [arXiv:1006.1311].

[10] A. Bazavov et al. [Fermilab Lattice and MILC], PoS LATTICE 2012, 159 (2012) [arXiv:1210.8431].

[11] M.A. Clark et al., Comput. Phys. Commun. 181, 1517 (2010) [arXiv:0911.3191]; R. Babich et al., [arXiv:1109.2935]. 